\title{
Reasoning about Intervals in Two Dimensions*
}

\author{
Björn Gottfried \\ Artificial Intelligence Group \\ Centre for Computing Technologies \\ University of Bremen, Germany \\ bg@tzi.de
}

\begin{abstract}
Qualitative reasoning approaches take on the challenge of dealing with commonsense knowledge in machines. This is important both to provide a means of efficient automatic reasoning and to overcome the differences between man and machine.

This paper proposes a new qualitative representation of spatial knowledge based on interval relations. The representation complements existing approaches by providing a new set of spatial relations. This allows qualitative reasoning about rigid objects, for example, in spatial configuration problems or in the context of spatiotemporal interactions between objects. The qualitative nature of this representation assists the engineering process and makes the behaviour of systems more easily comprehensible.
\end{abstract}

Keywords: Qualitative Spatial Reasoning, Knowledge Representation, Interval/Line Relations, Positional and Orientation Information

\section{Introduction}

Spatial reasoning is a fundamental prerequisite in a number of areas, including robotics, navigation, spatial planning, configuration, and high level vision. Qualitative spatial reasoning is distinguished by approaching problems in these fields while considering only those relations which are relevant either in a particular context, or from the point of view of cognitive systems, for example, by considering distinctions easily obtainable from perceptual processes. In this way it is possible to reason efficiently using small sets of relations which are just sufficient to solve problems at an abstract level.

We propose a generalisation of Allen's [1] onedimensional interval relations to two dimensions. Whereas Allen's approach focuses on reasoning about temporal knowledge, we aim at reasoning about spatial knowledge. In contrast to the one-dimensional case, additional variations of position and orientations of intervals are possible in two dimensions; and this allows for the investigation of the mutual dependence between location and direction. We propose a set of relations which represent those arrangements of objects which are easily distinguishable by perceptual processes. This is important in order both to analyse and to interpret sensor data efficiently. In particular, such relations can represent situations in which objects have an intrinsic orientation, or a direction of movement, or in which objects are seen from a special point of view. Imprecise and incomplete information about the relations between objects can be represented, and reasoning mechanisms are defined which allow for fine, as well as coarse, information. Without loss of generality, this can be demonstrated in the context of the following area of application.

Spatiotemporal interactions between objects are important in many applications. One example is the field of traffic supervision, which we shall use for demonstration purposes. In such a system a number of moving objects is observed; this has relevance for supervising crossroads, monitoring robots in push-button factories, or tracking objects generally.

We shall turn our attention towards an important subproblem: the integration of spatial knowledge which is obtained from different viewpoints. A single camera fails to comprehend a scene completely because objects often occlude each other. Many approaches avoid dealing with occlusion problems by assuming a bird's eye view from which a scene is surveyed completely. Such a viewpoint is difficult, frequently impossible, to obtain in practice. It is more realistic to take into account a number of cameras. These cameras have to collaborate in order to track robots, to avoid collisions, or to aid in navigation. We are consequently in need of mechanisms which can integrate spatial knowledge obtained from different viewpoints, as exemplified in Fig. 1. Here there are three objects, each represented by a vector. The left camera observes the relationship between $x$ and $y$, the right one between $y$ and $z$. Together, these cameras should be able to reach a conclusion regarding the relationship between $x$ and $z$.

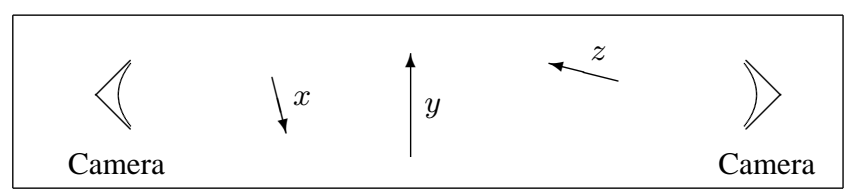

Figure 1: Observing a scene from two different viewpoints

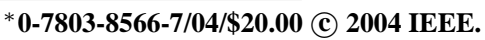




\section{Representing Intervals in the Plane}

We start by analysing which qualitative relations between intervals genuinely exist. In one dimension we are restricted to only two directions when changing the relative position of two intervals (Fig. 2.(c)). By this means we obtain the thirteen Allen relations (Fig. 2.(a)) describing qualitative relations between events in time. In the two-dimensional plane there are two more degrees of freedom concerning the arrangement of intervals. Firstly, it is possible to displace intervals not only horizontally, but also vertically, i.e. in relation to the second dimension (Fig. 2.(b)). Secondly, it is possible to change their orientation (Fig. 2.(d)). Any combination is admissible, leading to 225 positional relations (Fig. 3). Each such relation defines an equivalence class which subsumes infinitely many metrically distinguishable, but perceptually similar, relations. There are additional orientation variations: for example, relation number 17 in Fig. 3 allows all possible orientations within a range of $360^{\circ}$. But most positional relations do not allow all possible orientations. Relation number 1 in Fig. 3 allows exactly one orientation, relation number 2 allows orientation variations within a range of $90^{\circ}$. This shows the mutual dependence of location and direction.

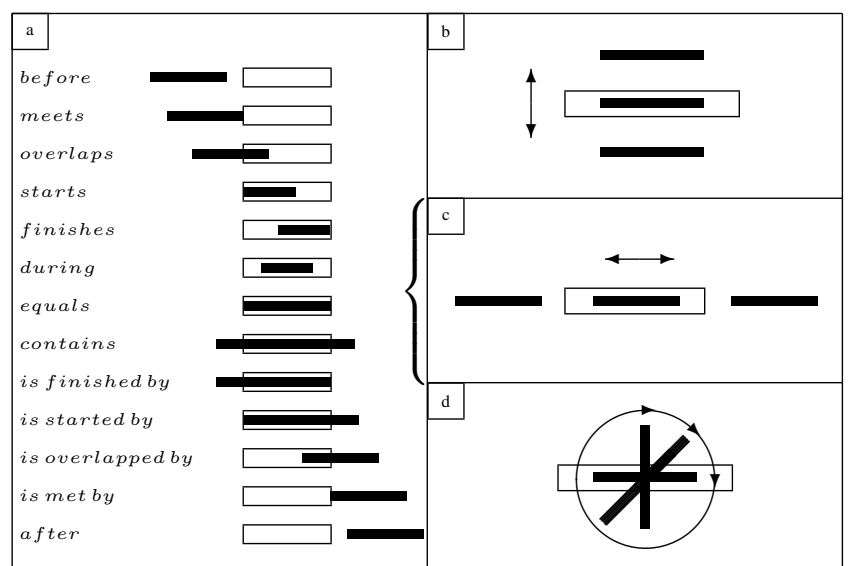

Figure 2: Left: Allen's thirteen one-dimensional interval relations; Right: Further possible variations in twodimensions; to distinguish the intervals, one interval is depicted as a white rectangle, the other one as a black rectangle

A representation scheme emerges when we relate two intervals in these ways. Fig. 4.(a) shows the one-dimensional case, Fig. 4.(b) the two-dimensional case. Such a reference system partitions the plane into different qualitative locations, defining possible positional relations. Embedding intervals in two dimensions suggests orthogonal reference systems, one induced by each interval. Orthogonal reference systems are appropriately related to the Euclidean plane and to what can be distinguished easily by perceptual processes. We, accordingly, partition the range of possible directions by $90^{\circ}$ angles, obtaining the 8 directions shown in Fig. 4.(c).
We refer to the interval which induces this reference system as the reference interval; the other interval being the primary interval - its position and orientation are described with respect to the reference interval. In order to distinguish these qualitative interval relations from precise metrical interval relations, we refer to them as to bipartite arrangements $-\mathcal{B A}$ for short. A single relation is denoted by $\mathcal{B} \mathcal{A}(i), i \in\{1,2, . ., 225\}$. Note that $\mathcal{B} \mathcal{A}$ concerns only positional relations. Describing interval $y$ with respect to interval $x$ it holds that $x_{y} \in \mathcal{B} \mathcal{A}$.

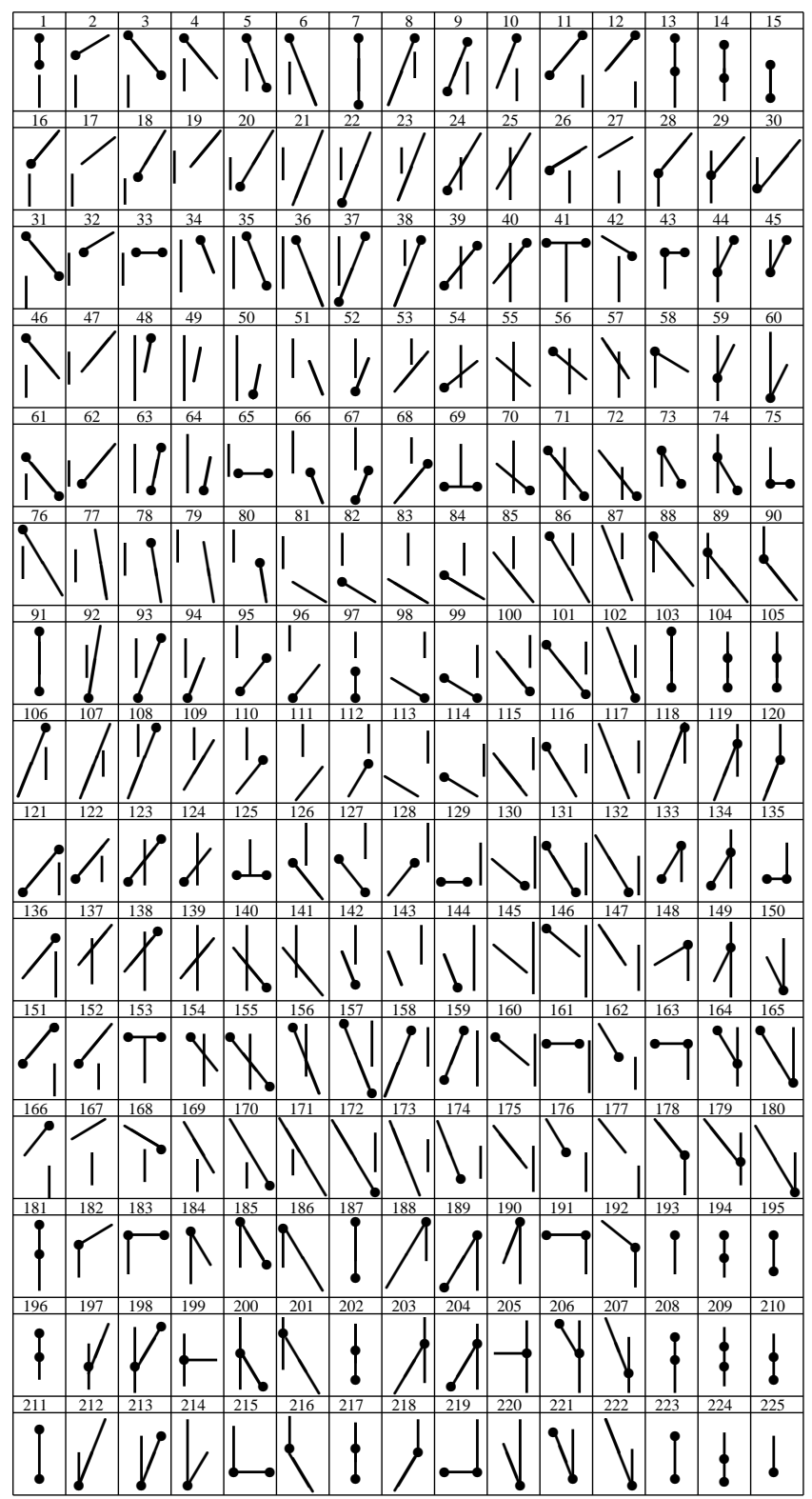

Figure 3: There exist 225 bipartite arrangements

\section{Partitioning $\mathcal{B A}$}

Considering the positional relations of $\mathcal{B A}$, as well as all orientation variations, we obtain quite a large set of relations. 
There exist 225 positional relations and 8 orientation variations, making together $225 * 8=1800$ relations. Each relation simultaneously encodes information about position and orientation. Since many relations, such as $\mathcal{B} \mathcal{A}(1)$ and $\mathcal{B} \mathcal{A}(2)$, allow only a subset of all orientation variations, there are altogether $225<n<1800$ relations.

Depending on the application area, some relations are more important than others. Relations, which are not to be distinguished anymore regarding the application at hand, can be merged into the same equivalence class. As a result a smaller set of coarser relations is obtained. Confining oneself to rather small sets of relations allows simpler modelling, more efficient reasoning, and easier comprehension of system behaviour.

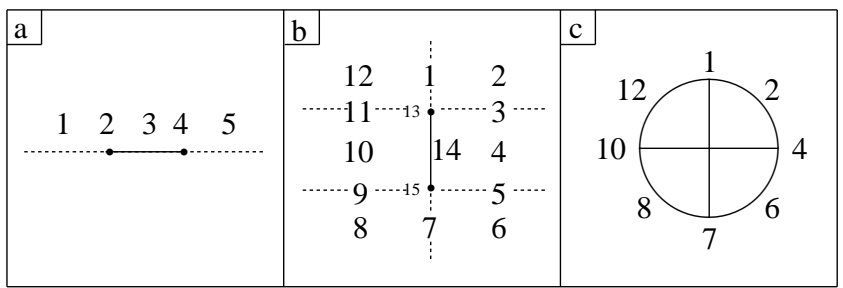

Figure 4: (a) The one-dimensional reference system distinguishes five positions - the reference interval runs from point 2 to 4; (b) the two-dimensional reference system distinguishes fifteen positions - the reference interval runs from point 13 to 15 ; (c) as for the locations different directions are defined by $90^{\circ}$ angles

Let us first of all consider a subset of $\mathcal{B} \mathcal{A}$ which is important irrespective of the application at hand. This subset contains those locations coinciding with the reference system, i.e. the locations $1,3,5,7,9,11,13,14,15$ in Fig. 4.(b). Endpoints of intervals lying at these singular locations are marked by bold points in Fig. 3. Whether the endpoints of primary intervals lie precisely at such locations is of no interest in the context of qualitative reasoning; it does not matter whether one object is exactly aligned with another. Instead, we want to restrict ourselves to relations which are just sufficient for a coarse representation. For this reason, we avoid endpoints lying in singular position: for example, $\mathcal{B} \mathcal{A}(2)$ is regarded as $\mathcal{B} \mathcal{A}(17)$, or as $\mathcal{B} \mathcal{A}(27)$ by excluding endpoints in singular positions or by including points in general positions - sometimes it is necessary to consider sets of both possibilities, for instance, when representing two parallel lines, which are equal in orientation and length; furthermore, relations such as $\mathcal{B} \mathcal{A}(1)$ are regarded as $\mathcal{B} \mathcal{A}(27)$, i.e. rather than considering singular locations neighbouring regions are considered that enclose that singular location. We are able to state, for example, that object $x$ is somewhere to the front left of object $y$, but it is not possible to state that object $x$ is precisely aligned with the left front of $y$. However, this is not actually the information we require. As we shall see later on, coarse positional information is already sufficient for solving several interesting problems.
We focus on problems dealing with rigid objects, and we have to restrict the set of possible relations accordingly. As a consequence we are interested in relations which are free of intersections, since rigid objects do not intersect each other. This allows us to omit relations such as $\mathcal{B A}(55)$.

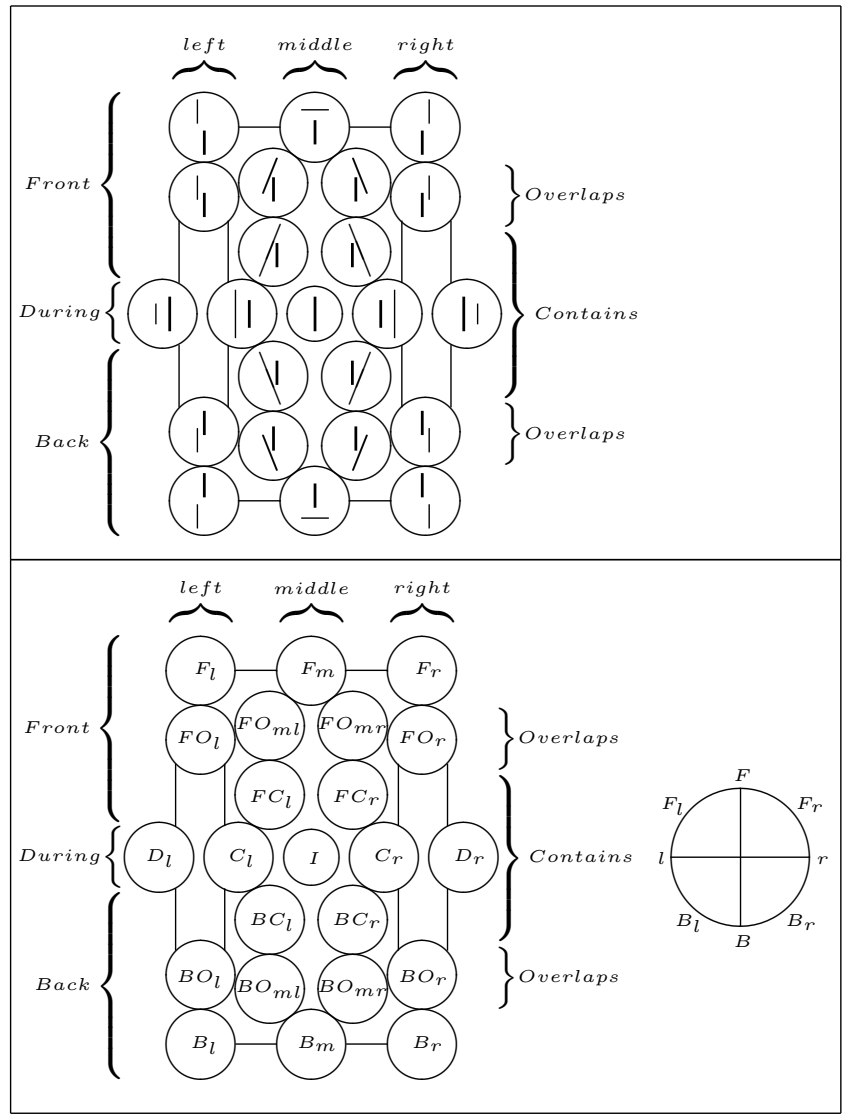

Figure 5: Top: Interval relations embedded in two dimensions, the vertical reference interval being displayed bold; Bottom: Left: Abbreviations for the relations; Right: the orientation variations for each relation

Putting together relations in this way, we eventually obtain the 23 relations shown in Fig. 5. Two relations are connected in this graph if they are conceptual neighbours. Accordingly to [6], two relations between pairs of intervals are conceptual neighbours, if they can be directly transformed into one another by continuously deforming, i.e. shortening, lengthening, or moving, the intervals. We refer to the set of these relations as $\mathcal{B A}_{23}$, and there is a mnemonic description of them at the bottom of Fig. 5 that aids in comprehending their meaning. The relations in $\mathcal{B A}_{23}$ are crucial for dealing with rigid objects. All conceivable arrangements between two rigid objects can be represented by $\mathcal{B A}_{23}$, since the relations in this set are jointly exhaustive regarding intersectionfree objects arranged in the plane. Moreover, each conceivable arrangement of two objects can be represented unambiguously by $\mathcal{B A}_{23}$, since these relations are pairwise disjoint. Taking into account all possible orientation variations we obtain 125 relations, which we refer to as $\mathcal{B} \mathcal{A}_{23}^{8}$, with the 


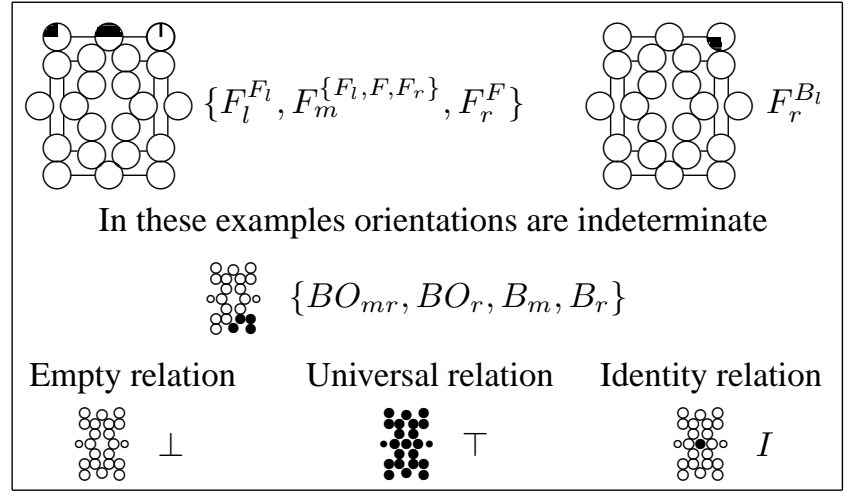

Figure 6: Examples for the iconic depiction

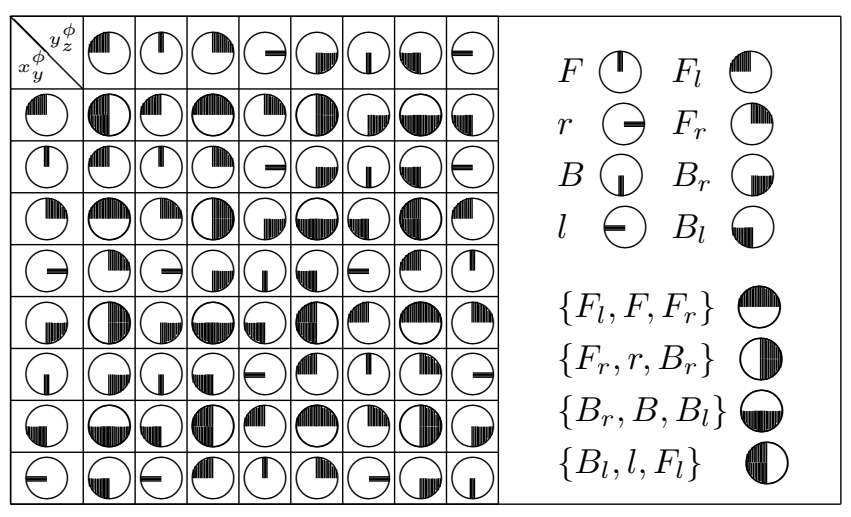

Figure 7: Given the orientation of $x_{y}$ and of $y_{z}$ possible orientations for $x_{z}$ are deduced

$y_{x}$. Fig. 8 shows the table of converse relations, $\Phi$ denoting the position, $\phi$ the orientation. For instance,

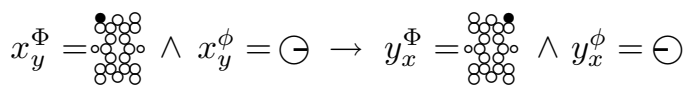
(first column, second row in Fig. 8). Note that Fig. 8 contains some empty sets which denote impossible combinations of position and orientation.

\subsection{Composition}

Finally, there is the composition operation. Given $x_{y}$ and $y_{z}$, we are interested in the relation $x_{z}$ which can be looked up in the composition table in which all possible transitivity relations are stored. Fig. 10 on the last page of this paper shows the composition table. For

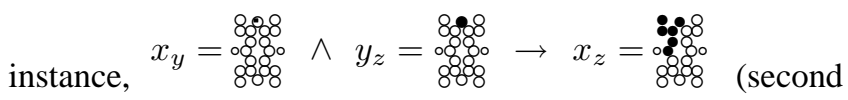
column, second row in the composition table). For the composition, we write $x_{z}=x_{y} \circ y_{z}$. The resulting orientation of $z$ with respect to $x$ is computed according to Fig. 7 .

If we have a set of intervals, the composition operation can be applied repeatedly to three-element subsets until no more relations can be updated. This results eventually in a consistent set of relations. If the updating process leads to an empty relation between two intervals an inconsistency has been recognised.

\subsection{Composition Table}

Let us discuss the composition operation more thoroughly. The 125 different basic relations of $\mathcal{B A}_{23}^{8}$ allow $125^{2}=$ 15625 compositions. We access the orientation of $y_{z}$ by $y_{z}^{\phi}$, and its position by $y_{z}^{\Phi} . y_{z}^{\phi}$ is irrelevant for the deduction of the position of $z$ with respect to $x$, since $x_{y}^{\phi}, x_{y}^{\Phi}$, and $y_{z}^{\Phi}$ sufficiently constrain how the position of $z$ is related to $x$; and the orientation of $z$ with respect to $x$ can be derived from the table in Fig. 7. Hence different orientation variations of $y_{z}$ can be pooled together in the composition table. Moreover, we can omit the identity relation since it behaves neutrally 


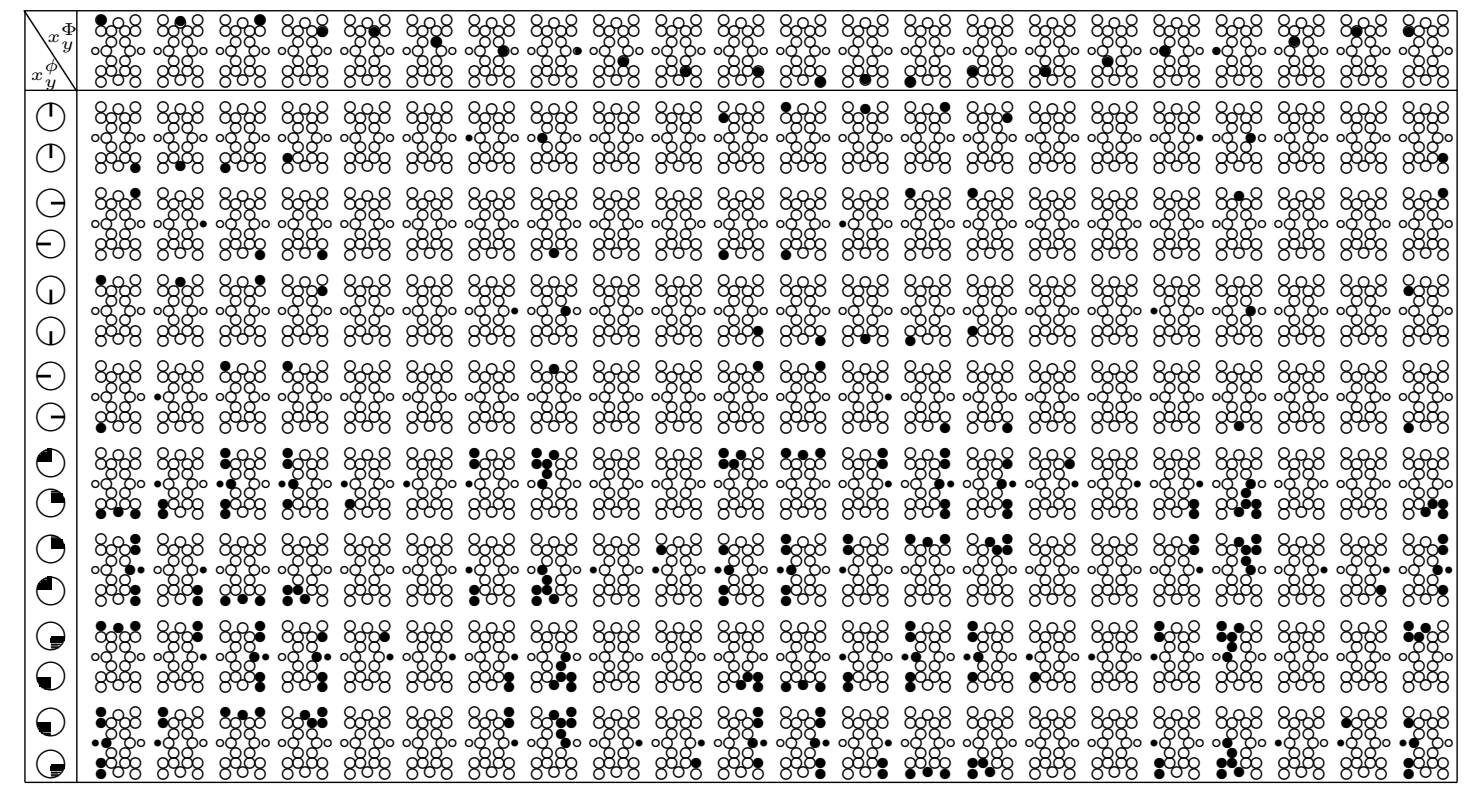

Figure 8: Converse relations: given $x_{y}$ in the upper row, $y_{x}$ is derived depending on the orientation of $y$ with respect to $x$ which determines the row; the orientation is given in the first column which also shows the change in orientation

with respect to composition. Furthermore, all compositions with the empty relation are empty; and all compositions with the universal relation result in the universal relation, except for the composition with the empty relation. As a consequence, there are only 22 possible relations to be considered for $y_{z}$, leading to $124 * 22=2728$ entries in the composition table. Exploiting the available symmetries we can reduce the composition table to $31 * 22=682$ entries. We shall refer to the composition table as $\mathcal{C} \mathcal{T}$. The following algorithm shows the computation of the 2728 compositions.

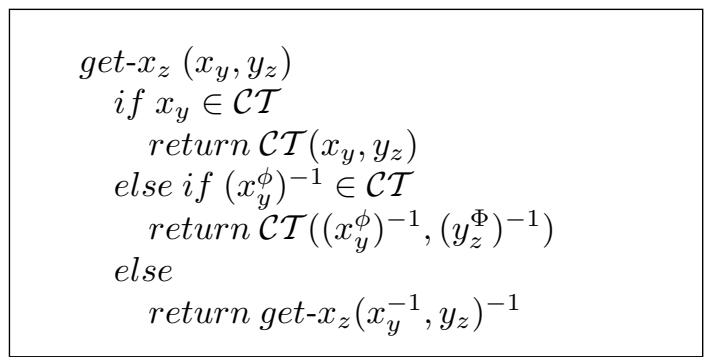

$\mathcal{C} \mathcal{T}\left(x_{y}, y_{z}\right)$ may simply be looked up in the composition table. $\left(x_{y}^{\phi}\right)^{-1}$ considers the inverse of the orientation of $y$ with respect to $x$ while keeping the position the same; the inverse of the orientation is a rotation by $180^{\circ} ;\left(y_{z}^{\Phi}\right)^{-1}$ can be expressed as $\left(\left(y_{z}+11\right) \bmod 22\right)$; this refers to the column in the composition table which is addressed by adding 11 columns to the column denoted by $y_{z}$; the modulo operator allows to return to the first column after the last column has been reached - this corresponds to a change in location by $180^{\circ} ; x_{y}^{-1}$ simultaneously takes the inverse of position and orientation, i.e. all occurrences of $B$ are changed into $F$ and vice versa, and all occurrences of $l$ are changed into $r$ and vice versa. The algorithm terminates at the latest after the second recursion step depending on the given position and orientation of $y$ with respect to $x$. Finally, Fig. 7 shows the combinations of two orientations in order to compute the composition of the orientation $x_{z}^{\phi}=x_{y}^{\phi} \circ y_{z}^{\phi}$. By considering these orientation combinations, the previous composition algorithm, and the identity relation, we obtain all 15625 compositions.

Two examples show the application of the composition algorithm:

$$
\begin{aligned}
& \text { 1. } x_{y}=F_{l}^{B_{l}} \text { and } y_{z}=F O_{r}^{F_{l}} \\
& \rightarrow x_{y}=F_{l}^{B_{l}} \notin \mathcal{C} \mathcal{T} \\
& \rightarrow\left(x_{y}^{\phi}\right)^{-1}=F_{l}^{F_{r}} \in \mathcal{C} \mathcal{T} \\
& \rightarrow \mathcal{C} \mathcal{T}\left(F_{l}^{F_{r}}, F O_{r}^{-1}\right)=\mathcal{C} \mathcal{T}\left(F_{l}^{F_{r}}, B O_{l}\right) \\
& \rightarrow x_{z}^{\Phi}=\left\{F_{l}, F O_{l}, C_{l}\right\} \\
& \rightarrow x_{z}^{\phi}=B_{l} \circ F_{l}=\left\{B_{r}, B, B_{l}\right\} \text { (Fig. 7) } \\
& \text { 2. } x_{y}=B_{m}^{B_{l}} \text { and } y_{z}=D_{l}^{r} \\
& \rightarrow x_{y}=B_{m}^{B_{l}} \notin \mathcal{C} \mathcal{T} \\
& \rightarrow\left(x_{y}^{\phi}\right)^{-1}=B_{m}^{F_{r}} \notin \mathcal{C} \mathcal{T} \\
& \rightarrow x_{y}^{-1}=F_{m}^{F_{r}} \in \mathcal{C} \mathcal{T} \\
& \rightarrow \rightarrow x_{z}^{-1}=\mathcal{C} \mathcal{T}\left(F_{m}^{F_{r}}, D_{l}\right)=\left\{F_{l}, F_{m}, F_{r}\right\} \text { (19th col.) } \\
& \rightarrow x_{z}^{\Phi}=\left(x_{z}^{-1}\right)^{-1}=\left\{B_{l}, B_{m}, B_{r}\right\} \\
& \rightarrow x_{z}^{\phi}=B_{l} \circ r=F_{l} \text { (Fig. 7) }
\end{aligned}
$$

All the entries in the composition table have been verified manually. Examining the composition table we can make some observations. (a) All compositions are valid. (b) There are comparatively few inferences with unique results; most conclusions contain disjunctions of possible results; such disjunctions represent coarse information. (c) Almost all disjunctions of alternative results form conceptual neighbourhoods. The only exceptions to this are disjunctions which are 
made up of different contains relations. They do not form a conceptual neighbourhood in every case since intersections are excluded; intersections would link different contains relations, such as $F C_{r}$ and $B C_{l}$, into neighbourhoods; see, for instance, row 3 and column 5 in $\mathcal{C} \mathcal{T}$. (d) In many cases neighbouring entries lead to similar neighbourhoods rather than to completely different neighbourhoods. But note that the composition table is not designed in such a way that all neighbouring preconditions form conceptual neighbours; for example, column seven, $C_{r}$, and column eight, $D_{r}$, do not form neighbouring concepts. (e) Only a small fraction of the elements of the power set $\mathcal{P}\left(\mathcal{B A}_{23}^{8}\right)$ appear in the composition table. (f) The composition table has only entries for atomic relations; for compound relations it is necessary to consider the unions of the compositions of the corresponding atomic relations. (g) In the complete table, with 15625 compositions, there are a lot of symmetries which we have used in order to reduce the number of entries necessary.

\section{Viewpoint Integration}

Now we shall turn to the problem of integrating spatial knowledge. In this context we are not only concerned with different views which we have to reconcile; these views are likely to be incomplete, inaccurate, or even erroneous, and we have to cope with such problems as well.

The relations in $\mathcal{B A}_{23}^{8}$ describe coarse spatial information. They allow us to cope with incompleteness by considering subsets of $\mathcal{B A}_{23}^{8}$ in which each element describes one possible situation. Incompleteness means that we do not have all the information regarding a scenario; we may characterise the scenario by considering all the situations that are possible for that scenario. If we know nothing, we have to take all possible situations into consideration, and our knowledge is completely undetermined. By contrast, if we are certain about the given scenario we can describe it using exactly one relation. The larger the subset we have to consider, the less we know. Imprecision is dealt with implicitly by the coarseness of these relations, and more imprecision can be dealt with again by subsets of $\mathcal{B A}_{23}^{8}$. Indeterminacy may also arise due to erroneous sensory information. We shall consider these issues in turn.

\subsection{Different Incomplete Viewpoints}

The arrangement of objects in Fig. 1 serves as a first example. The left camera observes $x$ with respect to $y$, and it holds that $y_{x}=D_{l}$. From the right camera we learn that $z_{y}=F_{l}$. We conclude, it holds that $z_{x}=z_{y} \circ y_{x}=F_{l} \circ D_{l}=F_{l}$. For the composition we need to know that the orientation of $y$ with respect to $z$ is $F_{r}$. The orientation of $x$ regarding $z$ is $F_{r} \circ B_{r}=\left\{B_{l}, B, B_{r}\right\}$, as shown in Fig. 7. This orientation cannot be computed precisely, although we can be certain that $x$ is somehow oriented backwards with respect to $z$.

\subsection{Different Imprecise Viewpoints}

It is sometimes not possible to determine the exact relation between objects. From a given point of view several different relations may be possible. Our observation is undetermined. Consider the left hand side of Fig. 9. The relation between $x$ and $y$ may be clear for both cameras. The camera at the bottom may decide for $y_{z}^{1}=\left\{C_{l}, F C_{l}\right\}$, since from its viewpoint it is not clear which side the front of $z$ is on with respect to $y$. By contrast, the other camera states that $y_{z}^{2}=\left\{C_{l}, F O_{l}, F_{l}\right\}$, since it is certain regarding the side of $z$ with respect to $y$; on the other hand it is not able to decide whether $z$ is completely in front of $y$ or whether it overlaps with $y$. The indeterminacy of each single camera is compensated for by the other camera; i.e. it holds that $y_{z}=y_{z}^{1} \cap y_{z}^{2}=\left\{C_{l}, F C_{l}\right\} \cap\left\{C_{l}, F O_{l}, F_{l}\right\}=C_{l}$.

\subsection{Different Erroneous Viewpoints}

Rather than being undetermined, observations may sometimes be erroneous. It is assumed that such erroneous relations are at least related to similar correct relations; that is, errors are similar to the right situation rather than completely wrong. The right hand side of Fig. 9 shows an example. In this case, there are two contradictory observations. Camera 1 observes $y_{z}^{1}=F O_{r}$, and camera 2 observers $y_{z}^{2}=F C_{r}$. All we can do is compute $x_{y}$ separately for each case. The results can then be compared. Relations which are part of both results may be the best candidates for the actual relationship. In the first case we obtain $x_{z}^{1}=\left\{F_{l}, F_{m}, F_{r}\right\}$; in the other case $x_{z}^{2}=\left\{F C_{l}, F_{m}, F C_{r}, F O_{m l}, F O_{m r}\right\}$. From what follows, $x_{z}=x_{z}^{1} \cap x_{z}^{2}=F_{m}$ may at least be close to the actual result.

\section{Related Work}

Different generalisations of [1] to the spatial domain have been proposed and surveyed by [2]. In contrast to these generalisations, which are based on point configurations ([7], [13]), regions ([3], [4]), or orthogonal projections of twodimensional objects on a number of different axes ([10], [11]), our new representation is based on intervals which are embedded in the two-dimensional plane. A few approaches deal with linear entities in two dimensions: line-line relations are found in [5] but these concern arbitrarily curved lines rather than straight intervals. [14] and [12] deal with straight line segments but they do not distinguish some visually salient arrangements of lines, as detailed by [8].

Our approach is a generalisation of [9] which is confined to special line arrangements that form polygons. Moreover, our approach is closely related to [7] in that we exploit the same reference system for describing the position of an in-

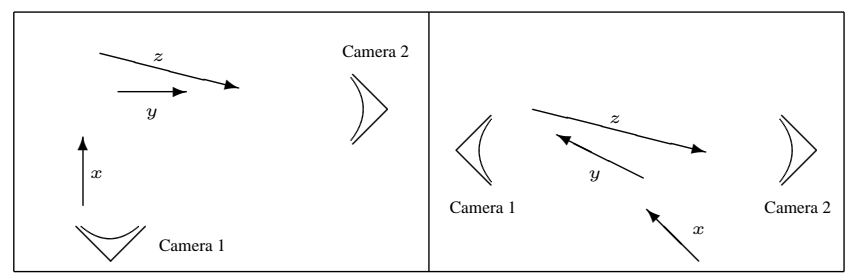

Figure 9: Two scenarios, each one is observed from two different viewpoints 
terval with respect to another interval as [7] uses to describe the position of a point relative to two other points. Further constraints arise from considering intervals rather than points. For example, though the qualitative positions of the endpoints of two intervals may be equal regarding a third interval, the two former intervals may lie on different sides with regard to the latter one. This is illustrated by the two relations denoted by Front-Contains-left and BackContains-right in Fig. 5.

In contrast to some approaches that are also based on intervals or respective line segments, as in [14] and [12], our relations are intersection-free; this is essential for modelling spatial scenes which deal with rigid objects. Furthermore, our approach allows additional distinctions concerning the arrangements of objects and their relative orientations. What our approach has in common with those mentioned above is the qualitative nature of variables and parameters, making systems less complex and more accessible to human users.

\section{Summary}

Our new approach advances current thinking in spatial reasoning, in that it provides a new set of relations. These relations are based on intervals and represent both positional and orientation information. By contrast, other approaches deal with points [7] and therefore they fail to describe, for instance, the overlap between objects; or they are based on regions [3], and describe relations between extended objects but fail to distinguish orientation information. Intervals can be considered as abstractions of objects, representing their intrinsic orientation, their direction of movement, or their extension seen from a particular viewpoint. The lengths of intervals may also represent the speed of objects or distances between objects. Whatever is represented by intervals, the proposed reasoning methods are always the same.

The concepts we have proposed are distinguished by their qualitative nature. This means that an object's position and orientation are defined relative to another object. Rather than using quantitative, external reference systems, we simply state whether an object is in front of another one, to the left of it, and so on. In this way we reason about the arrangement of a number of disconnected objects at an abstract level. This abstraction allows us to deal with imprecision by confining the relations used to distinctions which are perceptually clear — the other object is either on the left or on the right; we do not care about its exact position and we are not in need of precise sensor information. Incompleteness is dealt with by considering sets of possible relations; when knowledge is fragmentary we preclude those relations that we know cannot hold in a given context. All other relations are considered in a disjunctive way. As the reasoning procedures are the same for both complete and incomplete information this approach deals equally well with lateral views and top views; different views can even be reconciled as far as possible to identify objects uniquely. Such a qualitative representation can be comprehended very well indeed both from the engineer's and the user's points of view.

\section{References}

[1] J. F. Allen, "Maintaining knowledge about temporal intervals", CACM 26 (11), 832-843, 1983.

[2] A. G. Cohn and S. M. Hazarika, "Qualitative Spatial Representation and Reasoning: An Overview", Fundamenta Informaticae (43), pp. 2-32, 2001.

[3] D. A. Randell, Z. Cui and A. G. Cohn, "A spatial logic based on regions and connection", Proc 3rd Int. Conf. on Knowledge Representation and Reasoning, San Mateo, pp. 165-176, Morgan Kaufman, 1992.

[4] M. Egenhofer and R. Franzosa, "Point-set topological spatial relations", Int. Journal of Geographical Information Systems (5/2), pp. 161-174, 1991.

[5] M. Egenhofer, "Definitions of Line-Line Relations for Geographic Databases", 16th Int. Conf. on Data Engineering, 4046, 1993.

[6] C. Freksa, "Temporal reasoning based on semi-intervals", Artificial Intelligence (94), pp. 199-227, 1992.

[7] C. Freksa and K. Zimmermann, "On the Utilization of Spatial Structures for Cognitively Plausible and Efficient Reasoning”, IEEE Int. Conference on Systems, Man and Cybernetics, Chicago, pp. 261-266, October 1992.

[8] B. Gottfried, "Tripartite Line Tracks, Qualitative Curvature Information", Spatial Information Theory: Foundations of Geographic Information Science, Int. Conference COSIT 2003, W. Kuhn, M. Worboys and S. Timpf (eds.), Kartause Ittingen, 101117, LNCS (2825), Springer, September 2003.

[9] B. Gottfried, "Tripartite Line Tracks, Bipartite Line Tracks", KI 2003: Advances in Artificial Intelligence, A. Günter, R. Kruse and B. Neumann (eds.), Hamburg, 535-549, LNAI (2821), Springer, September 2003.

[10] H. Guesgen, "Spatial reasoning based on Allen's temporal logic", TR-89-049, Int. Computer Science Institute, Berkley, 1989.

[11] E. Jungert, "Symbolic spatial reasoning on object shapes for qualitative matching", Spatial Information Theory: Foundations of Geographic Information Science, Int. Conference COSIT 1993, A. U. Frank and L. Campari (eds.), Elba, 444462, LNCS(716), Springer, 1993.

[12] R. Moratz, J. Renz, and D. Wolter, "Qualitative Spatial Reasoning about Line Segments", ECAI 2000 Proceedings of the 14th European Conference on Artificial Intelligence, IOS Press, Amsterdam, 2000.

[13] C. Schlieder, "Ordering information and symbolic projection", Intelligent image database systems, S. K. Chang et al. (eds.), Singapore, 1995.

[14] C. Schlieder, "Reasoning about ordering", Spatial Information Theory: A Theoretical Basis for GIS, Int. Conference COSIT 1995, A. Frank and W. Kuhn (eds.), Semmering, 341349, LNCS(988), Springer, September, 1995.

Figure 10: Next page: the composition table 


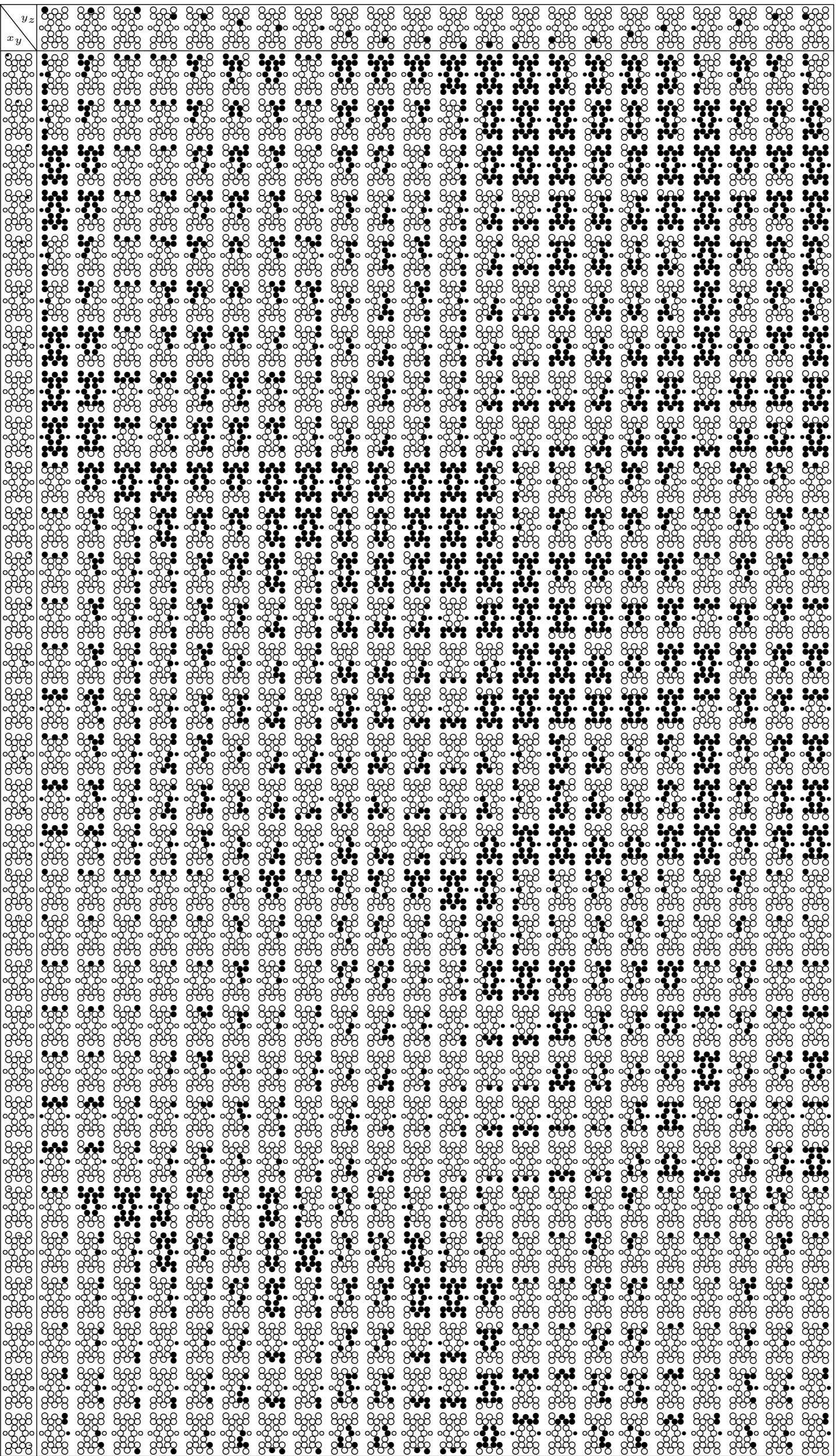

